\section{EXTRACELLULAR MATRIX PROTEOMICS IDENTIFIES MOLECULAR SIGNATURE OF SYMPTOMATIC CAROTID PLAQUES}

${ }^{1}$ Sarah R Langley, ${ }^{2}$ Karin Willeit, ${ }^{1}$ Athanasios Didangelos, ${ }^{3}$ Liubica Perisic Matic ${ }^{1}$ Philipp Skroblin, ${ }^{1}$ Javier Barallobre-Barreiro*, ${ }^{3}$ Mariette Lengquist, ${ }^{4}$ Gregor Rungger ${ }^{1}$ Alexander Kapustin, ${ }^{5}$ Ludmilla Kedenko, ${ }^{1}$ Ruifang Lu, ${ }^{6}$ Temo Barwari, ${ }^{1}$ Gonca Suna, ${ }^{1}$ Xiaoke Yin, ${ }^{7}$ Bernhard Iglseder, ${ }^{5}$ Bernhard Paulweber, ${ }^{2}$ Peter Willeit, ${ }^{8}$ Joseph Shalhoub, ${ }^{9}$ Gerard Pasterkamp, ${ }^{10}$ Claudia Monaco, ${ }^{3}$ Ulf Hedin, ${ }^{1}$ Catherine M. Shanahan, ${ }^{2}$ Johann Willeit, ${ }^{2}$ Stefan Kiechl Kielch, 'Manuel Mayr. ${ }^{1}$ King's College London; ${ }^{2}$ Department of Neurology, Medical University Innsbruck; ${ }^{3}$ Department of Molecular Medicine and Surgery, Vascular Surgery, Karolinksa University; ${ }^{4}$ Department of Neurology, Bruneck Hospital; ${ }^{5}$ First Department of Internal Medicine, Paracelsus Medical University; ${ }^{6}$ King's college London; ${ }^{7}$ Department of Geriatric Medicine, Paracelsus Medical University; ${ }^{8} / \mathrm{mperial}$ College; ${ }^{9}$ University Medical Centre Utrecht; ${ }^{10}$ Kennedy Institute, University of Oxford

10.1136/heartjinl-2017-311726.201

Introduction Recent findings have challenged the prevailing histology- or imaging-based definition of the vulnerable plaque.

Methods To investigate molecular characteristics associated with clinical instability of atherosclerosis, we performed a proteomics comparison of the vascular extracellular matrix and associated molecules in human carotid endarterectomy specimens from symptomatic versus asymptomatic patients. The proteomics data were integrated with gene expression profiling and an analysis of protein secretion by lipid-loaded human vascular smooth muscle cells.

Results The molecular signature of plaques from symptomatic patients identified by proteomics and at least one of the other two approaches comprised matrix metalloproteinase-9, chitinase-3-like protein 1, S100 calcium binding protein A8, S100 calcium binding protein $\mathrm{A}$, cathepsin $\mathrm{B}$, fibronectin and galectin-3-binding protein. Biomarker candidates were measured in 685 subjects of the Bruneck Study and found to be significantly associated with the progression to advanced atherosclerosis (as assessed by repeated carotid ultrasound) and the incidence of cardiovascular disease over a 10 year follow-up period. A 4-biomarker signature (matrix metalloproteinase-9, S100A8/S100A9, cathepsin D, and galectin-3-binding protein) improved risk prediction in terms of risk discrimination and classification and was successfully replicated in a second independent population (SAPHIR Study).

Conclusion Our study highlights the strength of tissue-based proteomics for biomarker discovery.

\section{THE CARDIAC HEPCIDIN/FERROPORTIN AXIS IS ESSNTIAL FOR CARDIAC IRON HOMEOSTASIS AND FUNCTION}

Samira Lakhal-Littleton*, Magda Wolna, Yu-Jin Chung, Helen Christian, Lisa Heather, Marcella Brescia, Vicky Ball, Rebeca Diaz, Ana Santos, Daniel Biggs, Kieran Clarke, Benjamin Davies, Peter Robbins. University of Oxford

\subsection{6/heartinl-2017-311726.202}

Background Iron deficiency and chronic heart failure are two of the most common disorders worldwide. Recent evidence has demonstrated that they are linked. Moreover, clinical trials have demonstrated the benefits of intravenous iron supplementation in chronic heart failure. However, cardiac iron homeostasis remains unexplored. Recently, our laboratory demonstrated that cardiac-specific deletion of the iron-exporting protein ferroportin causes fatal cardiac iron overload1. Ferroportin is known to be downregulated by the liver-derived hormone hepcidin. But hepcidin is also found in cardiomyocytes where its function remains unknown.

Methods and results To explore the function of cardiomyocyte hepcidin, we generated mice with a cardiomyocyte-specific deletion of hepcidin or with a cardiomyocyte-specific knock-in of a hepcidin-resistant ferroportin mutant. While both models maintain normal systemic iron homeostasis, they nevertheless develop cardiomyocyte metabolic dysfunction followed by fatal contractile impairment as a consequence of cardiomyocyte iron deficiency. Intravenous iron supplementation prevents both the development of metabolic dysfunction and contractile impairment. $^{2}$

Conclusions We conclude that regulation of iron export from cardiomyocytes by the cardiac hepcidin/ferroportin axis is essential to cardiomyocyte iron homeostasis and that its disruption leads to fatal cardiac dysfunction, even against a background of intact systemic iron homeostasis. These findings raise the possibility that hepcidin agonists/antagonists developed for disorders of systemic iron homeostasis could also modulate cardiac function.

\section{REFERENCES}

1. Lakhal-Littleton et al. An essential cell-autonomous role for hepcidin in cardiac iron homeostasis. Elife. 2016 Nov 29;5. pii: e19804. doi: 10.7554/eLife.19804.

2. Lakhal-Littleton et al. Cardiac ferroportin regulates cellular iron homeostasis and is important for cardiac function. PNAS, 2015;112, 3164-3169.

\section{ANALYSIS OF THE GPC6 GENE AND ITS INVOLVEMENT IN CARDIAC SEPTATION AND THE TETRALOGY OF FALLOT}

Gennadiy Tenin*. University of Manchester

\subsection{6/heartjnl-2017-311726.203}

Congenital heart diseases (CHD) are defects in the structure of the heart that are present at birth. They are amongst the most common birth defects and present in 9 out of 1000 births. Tetralogy of Fallot (ToF) is one of the most common $\mathrm{CHD}$ and is considered to be a multigenic condition as no single causative gene has been found so far. Studies on animal models have linked the development of TOF to defects in cardiac septation and heart valves formation. Our recent GWAS study found significant association of Tetralogy with Chr13q31 region ( 2 variants, $\mathrm{p}=7.4 \times 10^{-8}$ ) containing 2 poorly characterised genes, glypicans GPC5 and GPC6. Glypicans are transmembrane co-receptors involved in modulation of a number of signalling pathways. Available published data suggests that deletion of GPC5 has no effect on the embryonic development, while deletion of GPC6 may cause cardiac developmental abnormalities. We found that GPC6 is expressed exclusively in the mesenchymal cells (MC) populating endocardial cushions during critical stages of mouse and human cardiac septation, and later in the smooth muscle cells derived from these MC. We also found colocalisation of the Gpc6 with Pdgfra, suggesting novel GPC6 involvement in the regulation of Pdgf signalling pathway, required for cardiac septation, linking it to TOF defects. 